\title{
Solving Multiple Dataflow Queries Using WPDSs
}

\author{
Akash Lal ${ }^{1}$ and Thomas Reps ${ }^{1,2}$ \\ 1 University of Wisconsin; Madison, Wisconsin; USA. \{akash, reps\}@cs.wisc.edu \\ 2 GrammaTech, Inc.; Ithaca, NY; USA.
}

\begin{abstract}
A dataflow query asks for the set of reachable (abstract) states, given a starting set of states. In this paper, we show how to optimize multiple queries on the same program (each with a different starting set of states) for better overall running time. After a preprocessing phase, we obtain an asymptotic improvement in answering dataflow queries. We use weighted pushdown systems as the abstract model of a program. Our techniques are interprocedural. They are general, yet provide an impressive speedup. We applied our algorithm to three very different applications, one based on finding affine relations using linear algebra, and others for model checking Boolean programs, and obtained 1.5 -fold to 7 -fold speedups.
\end{abstract}

\section{Introduction}

Dataflow analysis is concerned with approximating program behavior. A dataflow query asks for the set of (abstract) program states (forward or backward) reachable from a given starting set of states, where a state is a (program location, data store) pair. One common application of (forward) dataflow analysis is to pose a single dataflow query from the initial state in which program execution starts. This produces an over-approximation of all program states that may arise during its execution. However, in certain situations, multiple dataflow queries need to be posed on the same program, each with a different starting set of states.

One such need arises in the analysis of concurrent programs, in the method presented in [13], which tracks program evolution for a bounded number of context switches. Here, a concurrent program consists of a set of threads that communicate via shared memory. For a thread $t$ of interest, the environment (consisting of the other threads) is only given control a fixed number of times. Each time, the environment can change the state of shared memory, thus affecting the execution of thread $t$. The analysis of such programs requires multiple dataflow queries to be posed on $t$. Whenever the environment changes the state of shared memory, a new query is posed on $t$, starting from this state

Multiple queries are also useful for program understanding, e.g., to find out the net effect of executing from one statement to another (to find dependences between them). Finding a loop summary for each loop is another example. Our applications ( $(5)$ are based on these examples. 
Answering multiple queries on the same program independently from each other usually involves repeated work. In this paper, we do preprocessing to compute certain basic facts about the program that can be reused each time a new dataflow query is posed. This improves the running time needed for answering multiple dataflow queries on the same program.

At the intraprocedural level, this work is inspired by our previous result [9], where we showed how to use Tarjan's path sequence algorithm [23], which computes regular expressions to represent a set of paths in a graph, to obtain a faster algorithm for answering a single dataflow query. In this paper, we show that the information computed by Tarjan's algorithm is also useful to avoid having to repeat fixpoint computations for answering multiple queries.

At the interprocedural level, a set of program paths can no longer be captured with a regular expression (the set may be a context-free language). We develop new techniques to address this complication: we show what preprocessing can be done to avoid recomputation across procedure boundaries, and how to isolate the intraprocedural computation to be able to use our intraprocedural algorithm.

Overall, with our techniques, the preprocessing is quite efficient, usually faster than solving two dataflow queries. After preprocessing, we obtain asymptotic improvements in answering each dataflow query (for programs whose control structure is mostly reducible), and only require iteration to a fixpoint when the starting set of states is infinite (i.e., in other cases, we do not need to go around program loops or recursive procedures). Our experiments show that this approach is advantageous even if as few as two queries need to be answered.

Our approach applies to any dataflow-analysis problem in which one has a domain of distributive dataflow-transfer functions closed under composition [22, $7]$. Some examples can be found in $[18,17,12]$. This paper mainly presents the work using the framework of weighted pushdown systems (WPDSs) [18], which generalize previous work on interprocedural analysis frameworks $[22,8,16]$. For details on how variants of the technique can be incorporated in solvers that work over control-flow graphs (ICFGs) see [10].

The contributions of this paper can be summarized as follows:

- We show how information computed by Tarjan's path sequence algorithm can be used to obtain asymptotic improvements in answering multiple intraprocedural queries ( $\S 3)$.

- We give a new WPDS reachability algorithm for answering interprocedural queries that carries over the above asymptotic improvements $(\S 4)$.

- We sketch variants of the technique that allow the ideas to be applied in other standard dataflow-analysis frameworks $(\S 4)$.

- We applied our techniques to three applications ( 55 ), and measured 1.5-fold to 7 -fold speedups over previous techniques, including optimized ones [9].

The rest of the paper is organized as follows: $\S 2$ gives background on WPDSs. $\S 3$ presents our algorithm for the intraprocedural case, and $\S 4$ generalizes it to the interprocedural case (WPDSs). $\S 5$ reports experimental results. $\S 6$ discusses related work. Proofs and other details can be found in [10]. 


\section{Program Model}

Definition 1. A pushdown system is a triple $\mathcal{P}=(P, \Gamma, \Delta)$ where $P$ is the set of states or control locations, $\Gamma$ is the set of stack symbols and $\Delta \subseteq P \times \Gamma \times P \times \Gamma^{*}$ is the set of pushdown rules. A configuration of $\mathcal{P}$ is a pair $\langle p, u\rangle$ where $p \in P$ and $u \in \Gamma^{*}$. A rule $r \in \Delta$ is written as $\langle p, \gamma\rangle \hookrightarrow\left\langle p^{\prime}, u\right\rangle$ where $p, p^{\prime} \in P, \gamma \in \Gamma$ and $u \in \Gamma^{*}$. These rules define a transition relation $\Rightarrow$ on configurations of $\mathcal{P}$ as follows: If $r=\langle p, \gamma\rangle \hookrightarrow\left\langle p^{\prime}, u\right\rangle$ then $\left\langle p, \gamma u^{\prime}\right\rangle \Rightarrow\left\langle p^{\prime}, u u^{\prime}\right\rangle$ for all $u^{\prime} \in \Gamma^{*}$. The reflexive transitive closure of $\Rightarrow$ is denoted by $\Rightarrow^{*}$.

Without loss of generality, we restrict PDS rules to have at most two stack symbols on the right-hand side [21]. The standard approach for modeling program control flow with a PDS is as follows: $P=\{p\}, \Gamma$ corresponds to program locations, and $\Delta$ corresponds to transitions in the interprocedural control-flow graph (ICFG) ${ }^{3}$ : A CFG edge $u \rightarrow v$ is modeled by a PDS rule $\langle p, u\rangle \hookrightarrow\langle p, v\rangle$; A call to procedure $g$ at location $l$ that returns to $r$ as $\langle p, l\rangle \hookrightarrow\left\langle p, g_{\text {enter }} r\right\rangle$; and a return from procedure $g$ as $\left\langle p, g_{\text {exit }}\right\rangle \hookrightarrow\langle p, \varepsilon\rangle$. In such an encoding, a PDS configuration $\left\langle p, \gamma_{1} \gamma_{2} \cdots \gamma_{n}\right\rangle$ stores the value of the program counter $\gamma_{1}$ and the stack of return addresses for unfinished calls as $\gamma_{2}, \gamma_{3}, \cdots, \gamma_{n}$ (in order).

A weighted PDS is obtained by supplementing a PDS with a weight domain that is a bounded idempotent semiring $[18,2]$. Such semirings are capable of encoding a number of abstractions [17]. WPDSs can encode the IFDS framework [16], and other dataflow analyses; see $[18,9]$ for more details.

Definition 2. A bounded idempotent semiring (or "weight domain") is a tuple $(D, \oplus, \otimes, \overline{0}, \overline{1})$, where $D$ is a set of weights, $\overline{0}, \overline{1} \in D$, and $\oplus$ (combine) and $\otimes$ (extend) are binary operators on $D$ such that

1. $(D, \oplus)$ is a commutative monoid with $\overline{0}$ as its neutral element, and where $\oplus$ is idempotent. $(D, \otimes)$ is a monoid with the neutral element $\overline{1}$.

2. $\otimes$ distributes over $\oplus$, i.e., for all $a, b, c \in D$ we have $a \otimes(b \oplus c)=(a \otimes b) \oplus(a \otimes c)$ and $(a \oplus b) \otimes c=(a \otimes c) \oplus(b \otimes c)$.

3. $\overline{0}$ is an annihilator with respect to $\otimes$, i.e., for all $a \in D, a \otimes \overline{0}=\overline{0}=\overline{0} \otimes a$.

4. In the partial order $\sqsubseteq$ defined by $\forall a, b \in D, a \sqsubseteq b$ iff $a \oplus b=a$, there are no infinite descending chains.

One may think of weights as dataflow transformers, extend as transformer composition, combine as meet, $\overline{0}$ as the transformer for an infeasible path, and $\overline{1}$ as the identity transformer.

The height $\mathcal{H}$ of a weight domain is defined to be the length of the longest descending chain in the semiring (if it exists). In this paper, we assume the height to be finite for ease of discussing complexity results. (For cases when the height is unbounded, the value $\mathcal{H}$ in the complexity results can be interpreted as the length of the longest descending chain that occurs while solving a particular problem instance, which is always bounded.)

${ }^{3} \mathrm{An}$ ICFG is a set of CFGs, one for each procedure, with additional edges going from a call-site to the entry node of the callee and from its exit node to the return site. 


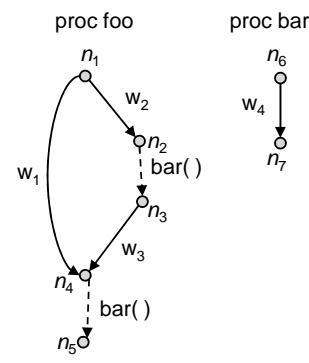

(1) $\left\langle p, n_{1}\right\rangle \hookrightarrow\left\langle p, n_{2}\right\rangle \quad w_{2}$

(2) $\left\langle p, n_{1}\right\rangle \hookrightarrow\left\langle p, n_{4}\right\rangle \quad w_{1}$

(3) $\left\langle p, n_{2}\right\rangle \hookrightarrow\left\langle p, n_{6} n_{3}\right\rangle \quad \overline{1}$

(4) $\left\langle p, n_{3}\right\rangle \hookrightarrow\left\langle p, n_{4}\right\rangle \quad w_{3}$

(5) $\left\langle p, n_{4}\right\rangle \hookrightarrow\left\langle p, n_{6} n_{5}\right\rangle \overline{1}$

(6) $\left\langle p, n_{6}\right\rangle \hookrightarrow\left\langle p, n_{7}\right\rangle \quad w_{4}$

(7) $\left\langle p, n_{7}\right\rangle \hookrightarrow\langle p, \varepsilon\rangle \quad \overline{1}$

Fig. 1. A program with two procedures and its corresponding WPDS. Procedure calls are represented using dashed arrows.

Definition 3. A weighted pushdown system is a triple $\mathcal{W}=(\mathcal{P}, \mathcal{S}, f)$ where $\mathcal{P}=(P, \Gamma, \Delta)$ is a pushdown system, $\mathcal{S}=(D, \oplus, \otimes, \overline{0}, \overline{1})$ is a bounded idempotent semiring and $f: \Delta \rightarrow D$ is a map that assigns a weight to each pushdown rule.

Let $\sigma=\left[r_{1}, \ldots, r_{k}\right] \in \Delta^{*}$ be a sequence of rules. We define $v(\sigma) \stackrel{\text { def }}{=} f\left(r_{1}\right) \otimes$ $\ldots \otimes f\left(r_{k}\right)$. Moreover, if for two configurations $c$ and $c^{\prime}$ of $\mathcal{P}, \sigma$ is a rule sequence that transforms $c$ to $c^{\prime}$, we say $c \Rightarrow^{\sigma} c^{\prime}$.

Definition 4. Let $\mathcal{W}=(\mathcal{P}, \mathcal{S}, f)$ be a WPDS, where $\mathcal{P}=(P, \Gamma, \Delta)$, and let $S, T \subseteq P \times \Gamma^{*}$ be sets of configurations. The interprocedural meet-over-allpaths (IMOP) value IMOP $(S, T)$ is defined as $\bigoplus\left\{v(\sigma) \mid s \Rightarrow^{\sigma} t, s \in S, t \in T\right\}$.

The IMOP value is the net transformation that occurs when going from one set of configurations to another. We write $\operatorname{IMOP}(s, t)$ for $\operatorname{IMOP}(\{s\},\{t\})$.

Fig. 1 shows how a program can be encoded using a WPDS. Each ICFG edge $e$ is encoded as a PDS rule whose weight is the dataflow transformer for $e$. More details on encoding programs as WPDSs can be found in $[18,17]$.

Model Semantics In WPDSs, program states are represented using weighted configurations, which are configuration-weight pairs. The pair $(c, w)$ describes the control state of the program as the PDS stack $c$, and the data state of the program using the weight $w$. A set of program states is represented by a function $\beta: P \times \Gamma^{*} \rightarrow D$, standing for the set $\left\{(c, \beta(c)) \mid c \in P \times \Gamma^{*}\right\}$. The set of all forward-reachable states starting from $\beta$ is the set poststar $(\beta)=\left\{\left(c^{\prime}, \oplus_{c}\{\beta(c) \otimes\right.\right.$ $\left.\left.\left.\operatorname{IMOP}\left(c, c^{\prime}\right)\right\}\right) \mid c^{\prime} \in P \times \Gamma^{*}\right\}$. In this case, we say that configuration $c^{\prime}$ can be reached with weight poststar $(\beta)\left(c^{\prime}\right)$ (which is $\overline{0}$ if $c^{\prime}$ is not reachable).

For example, the initial state of the program in Fig. 1 is $\left(\left\langle p, n_{1}\right\rangle, \overline{1}\right)$ and its reachable states include $\left(\left\langle p, n_{6} n_{3}\right\rangle, w_{2}\right)$ and $\left(\left\langle p, n_{6} n_{5}\right\rangle, w_{1} \oplus\left(w_{2} \otimes w_{4} \otimes w_{3}\right)\right)$.

\section{Solving Multiple Intraprocedural Queries}

Our interprocedural algorithm $(\S 4)$ will need to solve multiple intraprocedural queries. Thus, we address the latter case first. A directed graph is a special case of a PDS (no call or return rules). When a weight domain is paired with 
a directed graph, we obtain a model for intraprocedural analysis. To simplify the discussion of intraprocedural algorithms, we specialize some definitions to weighted graphs.

Definition 5. A weighted graph $G$ is a tuple $(V, E, \lambda)$, where $(V, E)$ is a directed graph, and $\lambda: E \rightarrow D$ is a function that labels each edge with a weight.

For vertices $v_{1}, v_{2} \in V$, a path $\sigma$ is defined as a sequence of edges that connect $v_{1}$ to $v_{2}$, in the standard way. In such a case, we say $v_{1} \Rightarrow^{\sigma} v_{2}$. The weight of a path $\sigma=\left[e_{1}, e_{2}, \cdots, e_{n}\right]$, written as $\lambda(\sigma)$, is defined to be $\lambda\left(e_{1}\right) \otimes \lambda\left(e_{2}\right) \otimes$ $\cdots \otimes \lambda\left(e_{n}\right)$. For sets of vertices $S, T \subseteq V$, the meet-over-all-paths (MOP) value is defined as the combine of weights of all paths that lead from a vertex in $S$ to a vertex in $T: \operatorname{MOP}(S, T)=\bigoplus\left\{\lambda(\sigma) \mid s \Rightarrow^{\sigma} t, s \in S, t \in T\right\}$. When $S=\{s\}$ and $T=\{t\}$ are singleton sets, we write $\operatorname{MOP}[s, t]$ as a shorthand for $\operatorname{MOP}(S, T)$.

Program states are vertex-weight pairs (vertices replace PDS configurations). Computing reachable states reduces to solving the following query:

Definition 6. Given a weighted graph $G$, and a set of vertices $S \subseteq V$ with a weight assignment $\mu: S \rightarrow D$, the IntraQ-query is to compute the weights $\operatorname{INTRAQ}_{G}(S, \mu)(v)=\bigoplus_{s \in S}\{\mu(s) \otimes M O P[s, v]\}$ for each $v \in V$.

$\operatorname{INTRA}_{G}(S, \mu)$ is the set of reachable states starting from $\{(s, \mu(s)) \mid s \in S\}$. We drop the subscript $G$ in IntraQ when it is obvious from the context.

When the graph $G$ is fixed, we can preprocess it to quickly answer subsequent queries. We now present three different algorithms for solving this query, where each of them trades off preprocessing time against time required to solve a query. Alg1: The first algorithm is the standard way of solving such queries using no preprocessing. It is a saturation algorithm: each vertex $v$ has a weight $l(v)$. Initially, $l(s)=\mu(s)$ for $s \in S$, and $\overline{0}$ for other vertices. Next, the rules $l(v):=$ $l(v) \oplus(l(u) \otimes \lambda(u, v))$ for each edge $(u, v)$ are used to update the weights until a fixpoint is reached. Then $l(v)$ is the required value for $\operatorname{InTRAQ}(S, \mu)(v)$. This requires time $O_{s}(|E| \mathcal{H})$, where $\mathcal{H}$ is the height of the weight domain, and the notation $O_{s}($.$) denotes the asymptotic number of semiring operations. (Because$ we consider weights as black boxes, the algorithms in this paper seek to minimize the number of semiring operations.)

The disadvantage of this method, which our other algorithms address, is that it requires a fixpoint computation to be performed; this is reflected in the cost by the dependence on the height $\mathcal{H}$ of the weight domain, which can be large.

Alg2: The second algorithm does the obvious preprocessing. It precomputes the values $\operatorname{MOP}\left[v_{1}, v_{2}\right]$ for each $v_{1}, v_{2} \in V$ by solving IntraQ $\left(\left\{v_{1}\right\},\left(v_{1} \mapsto \overline{1}\right)\right)$ for each $v_{1}$ using ALG1. Thus, preprocessing time is $O_{s}(|V||E| \mathcal{H})$. Once these MOP values are available, INTRAQ $(S, \mu)(v)$ can be solved from its definition in time $O_{s}(|S|)$. Thus, IntraQ $(S, \mu)$ can be solved in time $O_{s}(|S||V|)$, which is independent of $\mathcal{H}$. This may seem like the most efficient approach, but we show next that one can do better.

Consider the graph in Fig. 2. Suppose that $S=\left\{v_{2}, v_{3}\right\}, \mu\left(v_{2}\right)=w_{2}$, and $\mu\left(v_{3}\right)=w_{3}$. Then ALG2 would require approximately $2|V|$ semiring operations 
because it considers $v_{2}$ and $v_{3}$ separately from each other. However, notice that vertex $v_{4}$ dominates all other vertices with respect to $v_{2}$ and $v_{3}$, i.e., any path in the graph starting at $v_{2}$ or $v_{3}$ must pass through $v_{4}$ before reaching vertices $v_{5}$ to $v_{k}$ (and vertex $v_{1}$ is unreachable). Based on this observation, we can prove that $\operatorname{IntraQ}(S, \mu)\left(v_{i}\right)=\operatorname{Intra} Q(S, \mu)\left(v_{4}\right) \otimes \operatorname{MOP}\left[v_{4}, v_{i}\right]$ for $v_{i} \in\left\{v_{5}, \cdots, v_{k}\right\}$ Therefore, we only need to compute $\operatorname{InTRAQ}(S, \mu)\left(v_{4}\right)$ and other values can follow from this value using just one operation. This method would only require, approximately, $|V|$ number of operations.

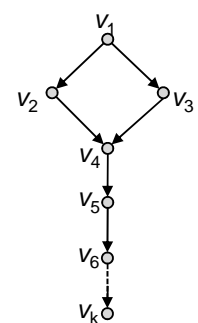

Fig. 2. A graph

This observation can be generalized to say that the weight on a vertex should be computed before the weights on vertices dominated by it are computed. This has been already captured nicely by Tarjan's algorithm [23] to solve path problems on graphs. However, it has only been used in the context of solving a single query, which we generalize to multiple queries. First, we summarize the essential details of Tarjan's algorithm.

Definition 7. A path expression is a regular expression over the edges of a graph defined using the following grammar: $r:=\emptyset|\varepsilon| e\left|r_{1} . r_{2}\right| r_{1} \cup r_{2} \mid r^{*}$ where $e$ is an edge in the graph. A path expression $r$ is said to represent the set of paths in the language $\mathcal{L}(r)$ of $r$ when interpreted as a regular expression. Furthermore, a path expression is said to be of type $(u, v)$ if all paths in $\mathcal{L}(r)$ go from vertex $u$ to vertex $v$.

For example, for the graph in Fig. 2, the expression $\left(\left(e_{12} \cdot e_{24} \cup e_{13} \cdot e_{34}\right) \cdot e_{45}\right)$, where $e_{i j}$ is the edge $\left(v_{i}, v_{j}\right)$, denotes the set of all paths from $v_{1}$ to $v_{5}$, and is of type $\left(v_{1}, v_{5}\right)$.

We extend $\lambda$ to path expressions as follows: $\lambda(\emptyset)=\overline{0}, \lambda(\varepsilon)=\overline{1}, \lambda\left(r_{1} . r_{2}\right)=$ $\lambda\left(r_{1}\right) \otimes \lambda\left(r_{2}\right), \lambda\left(r_{1} \cup r_{2}\right)=\lambda\left(r_{1}\right) \oplus \lambda\left(r_{2}\right)$, and $\lambda\left(r^{*}\right)=\lambda(r)^{*}$. Here, we define the weight $w^{*}$ as the infinite combine $\overline{1} \oplus w \oplus(w \otimes w) \oplus(w \otimes w \otimes w) \oplus \ldots$, which exists because of Defn. 2(item 4). One can show that $w^{*}=(\overline{1} \oplus w)^{\mathcal{H}}$, and calculate it using repeated squaring in time $O_{s}(\log \mathcal{H})$. Consequently, the following lemma holds. (We define $|r|$ to be the length of the expression.)

Lemma 1. For a path expression $r$ and $\lambda$ defined as above, $\lambda(r)=\bigoplus\{\lambda(\sigma) \mid$ $\sigma \in \mathcal{L}(r)\}$. Moreover, it can be calculated in time $O_{s}(|r| \log \mathcal{H})$.

Tarjan's algorithm is based on computing path expressions to represent the set of paths between each pair of vertices. However, instead of computing a separate path expression for each pair of vertices, it computes a path sequence, which is a more concise way of representing all paths in a graph.

Definition 8. A path sequence of a directed graph $G=(V, E)$ is a sequence $\left(r_{1}, u_{1}, v_{1}\right),\left(r_{2}, u_{2}, v_{2}\right), \cdots\left(r_{k}, u_{k}, v_{k}\right)$, where $u_{i}, v_{i} \in V, r_{i}$ is a path expression of type $\left(u_{i}, v_{i}\right)$ such that for any nonempty path $\sigma$ in $G$, there is a sequence of indices $1 \leq i_{1}<i_{2}<\cdots<i_{l} \leq k$ and a partition of $\sigma$ into nonempty paths $\sigma=\sigma_{1} \sigma_{2} \cdots \sigma_{l}$ and $\sigma_{j} \in \mathcal{L}\left(r_{i_{j}}\right)$ for all $1 \leq j \leq l$. 
Fig. 3(a) is an algorithm that uses a path sequence to create path expressions $r[s, v]$ that represent the set of all paths from $s$ to $v$, for each $v \in V$ and a fixed $s \in V$ [23]. Using Lemma 1 , we get $\operatorname{MOP}[s, v]=\lambda(r[s, v])$. Equivalently, the path expressions can be evaluated first and then put to-

\begin{tabular}{|c|c|}
\hline $\begin{array}{l}\text { 1: // initialize } \\
2: \text { for all } v \in V \text { do } \\
3: \quad r[s, v]:=\emptyset \\
4: \text { end for } \\
5: r[s, s]:=\varepsilon \\
6: \quad / / \text { solve } \\
\text { 7: for } i=1 \text { to } k \text { do } \\
8: \quad r\left[s, v_{i}\right]:=r\left[s, v_{i}\right] \cup \\
\quad\left(r\left[s, u_{i}\right] . r_{i}\right) \\
\text { 9: } \text { end for }_{(a)}^{(a)}\end{array}$ & $\begin{array}{l}\text { 1: } / / \text { initialize } \\
\text { 2: for all } v \in V \text { do } \\
\text { 3: } \quad \operatorname{MOP}[s, v]:=\overline{0} \\
\text { 4: end for } \\
\text { 5: } \operatorname{MOP}[s, s]:=\overline{1} \\
\text { 6: } / / \text { solve } \\
\text { 7: for } i=1 \text { to } k \text { do } \\
\text { 8: } \quad \operatorname{MOP}\left[s, v_{i}\right]:=\operatorname{MOP}\left[s, v_{i}\right] \\
\quad \oplus\left(\operatorname{MOP}\left[s, u_{i}\right] \otimes \lambda\left(r_{i}\right)\right) \\
\text { 9: end for }(\mathrm{b})\end{array}$ \\
\hline
\end{tabular}

Fig. 3. Computing MOP values using the path sequence $\left\{\left(r_{i}, u_{i}, v_{i}\right)\right\}_{i=1}^{k}$. gether to get the MOP weights, as shown in Fig. 3(b).

Tarjan's algorithm computes a path sequence for a graph in time $O(|E| \log |V|+$ $\delta$ ), where $\delta$ is a term that denotes the irreducibility factor of the graph. For reducible graphs, $\delta=0$ and, in general, $\delta$ is bounded by $|V|^{3}$. Because the graphs we work with come from CFGs of procedures, they are mostly reducible and the $\delta$ term can be ignored (which is confirmed by our experiments). Evaluating all path expressions takes time $O_{s}((|E| \log |V|+\delta) \log \mathcal{H})$. After that, given a vertex $s$, solving for $\operatorname{MOP}[s, v]$ for all vertices $v$ requires time $O_{s}(|E| \log |V|+\delta)$, which is almost linear in the size of the graph. We ignore $\delta$ in the rest of the paper.

Alg3: Suppose that we wish to solve $\operatorname{IntraQ}(S, \mu)$ on $G=(V, E, \lambda)$. We construct a new graph $G^{\prime}=\left(V^{\prime}, E^{\prime}, \lambda^{\prime}\right)$ by adding a new vertex to $G$ : for some $v_{0} \notin V, V^{\prime}=V \cup\left\{v_{0}\right\}, E^{\prime}=E \cup\left\{\left(v_{0}, s\right) \mid s \in S\right\}, \lambda^{\prime}=\lambda \cup\left\{\left[\left(v_{0}, s\right) \mapsto \mu(s)\right] \mid\right.$ $s \in S\}$. Then $\operatorname{MOP}_{G^{\prime}}\left[v_{0}, v\right]=\operatorname{InTRAQ}_{G}(S, \mu)(v)$. Thus, we need to compute MOP values on $G^{\prime}$. This trick is similar to the standard one of reducing a multisource reachability problem to a single-source reducibility problem. The following observation shows that a path sequence for $G^{\prime}$ can be computed from that of $G$ :

Lemma 2. If $p s$ is a path sequence of $G$, then by concatenating the sequence $\left\{\left(v_{0}, s,\left(v_{0}, s\right)\right) \mid s \in S\right\}$ (with any arbitrary order chosen to enumerate vertices in $S)$ in front of ps, one obtains a path sequence for $G^{\prime}$.

The preprocessing step of ALG3 computes a path sequence for $G$ and evaluates the weight of each of its path expressions. Then to solve each query, Alg3 uses the path sequence for $G^{\prime}$, constructed using Lemma 2 , as input to the algorithm in Fig. $3(b)$. This gives us the required weights $\operatorname{InTRAQ}_{G}(S, \mu)(v)$ as $\mathrm{MOP}_{G^{\prime}}\left[v_{0}, v\right]$. AlG3 requires $O_{s}(|E| \log |V| \log \mathcal{H})$ preprocessing time and $O_{s}(|S|+|E| \log |V|)$ time to solve each query. This is much better than Alg2 because for CFGs, $|E|$ is usually $O(|V|)$. We used ALG3 in our experiments.

\section{Solving Multiple Queries on WPDSs}

For graphs, the number of vertices is finite, but for WPDSs, the number of configurations may be infinite (when the program is recursive), or very large 
(exponential in the number of procedures when not recursive). For this reason, sets of weighted configurations (or program states) are represented symbolically using weighted automata [18].

Definition 9. A weighted automaton $\mathcal{A}$ is a finite-state automaton where each transition is additionally labeled with a weight. The weight of a path in the automaton is obtained by taking an extend of the weights on the transitions in the path in the backward direction. The automaton is said to accept a configuration $\langle p, u\rangle$ with weight $w$, denoted by $\mathcal{A}(\langle p, u\rangle)$, if $w$ is the combine of weights of all accepting paths for $u$ starting from state $p$ in $\mathcal{A}(w=\overline{0}$ if $u$ is not accepted). The set of states of $\mathcal{A}$ is assumed to contain $P$, the set of PDS states.

A weighted automaton $\mathcal{A}$ represents the set of states $\mathcal{R}(\mathcal{A})=\{(c, \mathcal{A}(c)) \mid$ $\mathcal{A}(c) \neq \overline{0}\}$. An important result is that the set $\operatorname{poststar}(\mathcal{R}(\mathcal{A})$ ) (as defined in $\S 2)$ can also be represented by a weighted automaton [18]. For brevity, we call such an automaton poststar $(\mathcal{A})$. Our goal is to preprocess a given WPDS so that $\operatorname{poststar}(\mathcal{A})$ can be computed quickly for any given $\mathcal{A}$.

An example is shown in Fig. 4(a). Note how the weight for a configuration $\left\langle p, n_{7} n_{3}\right\rangle$ is represented in a compositional way in the automaton. Procedure bar is analyzed independently of its calling context, resulting in weight $w_{4}$ for transition $\left(p, n_{7}, q\right)$. The weight $w_{2}$ at the call site $n_{3}$ to bar is captured on the transition $\left(q, n_{3}, a c c\right)$, resulting in a total weight of $w_{4} \otimes w_{2}$ for $\left\langle p, n_{7} n_{3}\right\rangle$. We will use the fact that procedures are analyzed independently of their calling context (also customary in most summary-based interprocedural analyses) in our favor. (As we shall see later, this implies that weights have to be propagated from a procedure to its callers, but not to procedures that it calls.)

Fix $\mathcal{W}=((P, \Gamma, \Delta), \mathcal{S}, f)$ to be a WPDS. Fix $\mathcal{A}_{\text {start }}$ to be the input query automaton, for which we want to compute $\operatorname{poststar}\left(\mathcal{A}_{\text {start }}\right)$. To simplify the discussion, assume that $\mathcal{W}$ was created from a program as described in $\S 2$, and $P=\{p\}$ is a singleton set (our implementation handles any WPDS, however).

Preprocessing ( $i$ ) First, we compute a summary for each procedure. (For a procedure starting at node $e$, it is defined as $\operatorname{IMOP}(\langle p, e\rangle,\langle p, \varepsilon\rangle))$. Using these summaries, we construct a weighted graph for each procedure from its CFG: the call edges (from call site to return site) are replaced with a summary of the called procedure. For $\gamma \in \Gamma$, let $\mathrm{PR}_{\gamma}$ be the procedure that contains $\gamma, G_{\gamma}$ be the weighted graph for $\mathrm{PR}_{\gamma}, e_{\gamma}$ its unique entry node, and $x_{\gamma}$ its unique exit node. (Note that $\mathrm{MOP}_{G_{\gamma}}\left[e_{\gamma}, x_{\gamma}\right]$ also equals the summary for $\mathrm{PR}_{\gamma}$.) Next, for each weighted graph $G$ of a procedure, we compute: (ii) its path sequence (preprocessing for ALG3) and (iii) values $\operatorname{MOP}_{G}\left[\gamma, x_{\gamma}\right]$ and $\operatorname{MOP}_{G}\left[e_{\gamma}, \gamma\right]$ for each node $\gamma$ of the procedure.

The procedure summaries can be computed using standard algorithms, after which the path sequences can be constructed using Tarjan's algorithm. This would be an acceptable solution, but we can do better. We use our techniques from [9] to compute both of these at the same time. Briefly, the call-return edge in the CFG of a procedure is labeled with a variable whose value stands for the (as yet uncomputed) summary of the called procedure. Then the procedure summary is represented using a path expression (from entry node to return node) 


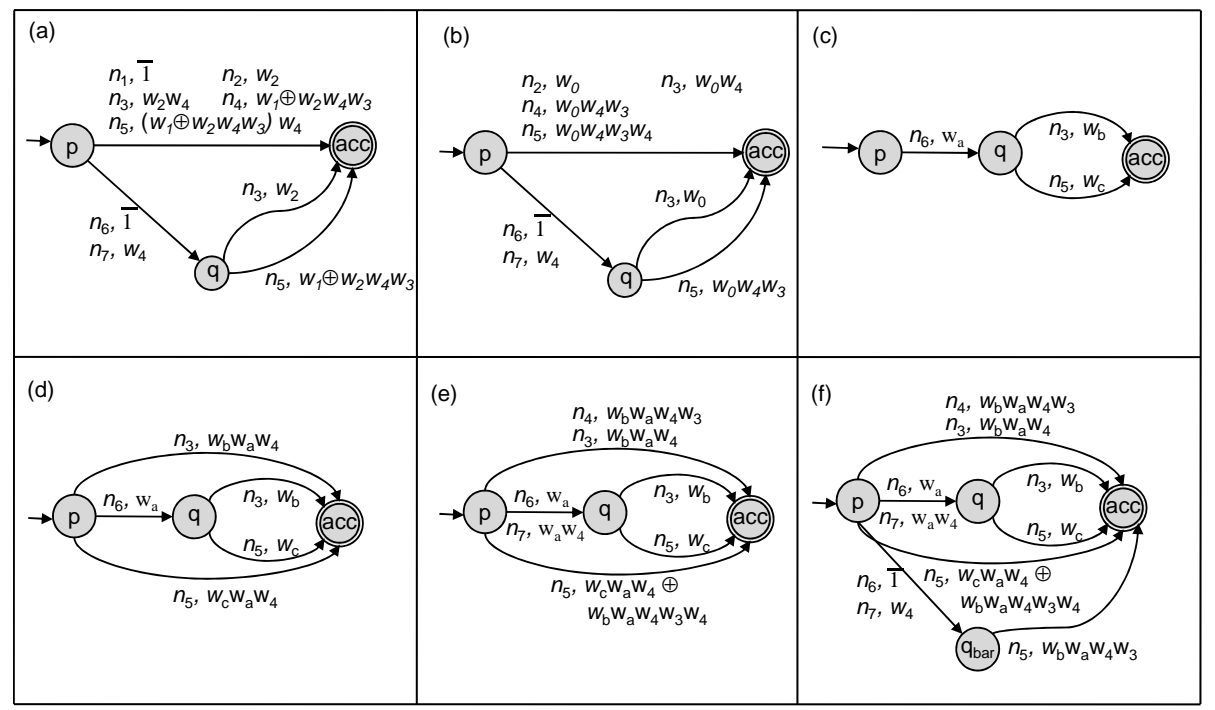

Fig. 4. Various automata related to the WPDS of Fig. 1. In all the weighted automata, juxtaposition of weights denotes their extend, acc is the accepting state, and parallel transitions have sometimes been collapsed into a single edge. Labels on transitions are (stack symbol, weight) pairs. (a) An automaton for poststar $\left(\left\{\left(\left\langle p, n_{1}\right\rangle, \overline{1}\right)\right\}\right)$. (b) An automaton for poststar $\left(\left\{\left(\left\langle p, n_{2}\right\rangle, w_{0}\right)\right\}\right)$. (c) Automaton $\mathcal{A}_{\text {start }}$. (d) Automaton $\mathcal{A}_{\text {pop }}$ obtained after running the pop-phase.(e) Automaton $\mathcal{A}_{\text {int }}$ created while running the growth phase on $\mathcal{A}_{\text {pop }}$. $(f)$ The final result of running the growth phase on $\mathcal{A}_{\text {pop }}$.

computed from its path sequence. This expression will have variables standing for summaries of called procedures. This gives rise to a set of equations whose solution solves for all summaries. In [9], we showed that this technique provides up to 5 times speedup over standard algorithms for computing procedure summaries, and we obtain a path sequence as a by-product. The path sequences can then be used to quickly compute the required MOP values for (iii) [23].

ICFG-version Before describing how our algorithm works with weighted automata, we briefly describe how it would work with ICFGs (after preprocessing). (Full details are in [10].) Suppose that we are given a set $R$ of node-weight pairs (starting states), where the nodes may be from multiple procedures, and we want to calculate the reachable set of node-weight pairs.

One challenge is to isolate the intraprocedural work. An INTRAQ query on a set $S=\left\{s_{1}, \cdots, s_{k}\right\}$ can also be solved by making a separate query for each $s_{i}$ and taking a combine of the results, but this is far less efficient than making a single query on $S$. Thus, we want to minimize the number of INTRAQ queries made for each procedure. For example, for the program in Fig. 1, suppose $R=$ $\left\{\left(n_{6}, w_{a}\right),\left(n_{4}, w_{b}\right)\right\}$. Then the pair $\left(n_{6}, w_{a}\right)$ can produce the pairs $\left(n_{3}, w_{a} \otimes w_{4}\right)$ and $\left(n_{5}, w_{a} \otimes w_{4}\right)$ inside foo, when bar returns. We would then like to make just one INTRAQ query on foo with $S=\left\{n_{3}, n_{4}, n_{5}\right\}$ (and appropriate weights), 


$$
\begin{aligned}
& \left\langle p, \gamma_{1} \gamma_{2} \gamma_{3} \cdots \gamma_{n}\right\rangle \Rightarrow^{\sigma_{1}} \quad\left\langle p, \gamma_{2} \gamma_{3} \cdots \gamma_{k+1} \gamma_{k+2} \cdots \gamma_{n}\right\rangle \\
& \Rightarrow^{\sigma_{2}} \quad\left\langle p, \gamma_{3} \cdots \gamma_{k+1} \gamma_{k+2} \cdots \gamma_{n}\right\rangle \\
& \Rightarrow^{*} \quad \cdots \\
& \Rightarrow^{\sigma_{k}} \quad\left\langle p, \gamma_{k+1} \gamma_{k+2} \cdots \gamma_{n}\right\rangle \\
& \Rightarrow^{\sigma_{k+1}}\left\langle p, u_{1} u_{2} \cdots u_{j} \gamma_{k+2} \cdots \gamma_{n}\right\rangle
\end{aligned}
$$

Fig. 5. A path in the PDS's transition relation; $u_{i} \in \Gamma, j \geq 1, k \leq n, \sigma_{h} \in \Delta^{*}$.

instead of making a query with just $n_{4}$ first, and then realizing that the procedure has to be explored again from $n_{3}$ and $n_{5}$.

The algorithm proceeds in two phases. The first phase moves across procedure boundaries: if $(n, w) \in R$ then we propagate this weight to the callers of $\mathrm{PR}_{n}$. We add $\left(r, w \otimes \mathrm{MOP}_{G_{n}}\left[n, x_{n}\right]\right)$ to $R$ for each return site $r$ of calls to $\mathrm{PR}_{n}$ (if the pair $\left(r, w^{\prime}\right)$ was already present in $R$, then change $w^{\prime}$ to $\left.w^{\prime} \oplus\left(w \otimes \mathrm{MOP}_{G_{n}}\left[n, x_{n}\right]\right)\right)$. This continues until saturation. The use of (precomputed) $\mathrm{MOP}\left[n, x_{n}\right]$ weights allow us to quickly jump from a procedure to its callers.

The second phase is intraprocedural. If $\left(n_{1}, w_{1}\right), \cdots,\left(n_{k}, w_{k}\right) \in R$ and the $n_{i}$ are from the same procedure, run IntraQ $\left(\left\{n_{1}, \cdots, n_{k}\right\},\left[n_{i} \mapsto w_{i}\right]\right)$ to get weights on all other nodes in the procedure. This is repeated for all procedures. The resulting node-weight pairs represent all reachable states.

The extension of these ideas to WPDSs have two complications: First, configurations add constraints on how weights get propagated to callers. For example, starting at configuration $\left\langle p, \gamma_{1} \gamma_{2}\right\rangle$ constrains weight propagation to $\gamma_{2}$ when $\mathrm{PR}_{\gamma_{1}}$ returns (and not to its other return sites). Second, the number of configurations may be infinite, forcing us to use automata-based symbolic representations.

The above ICFG version only required at most one INTRAQ query per procedure, which is ideal. The general version for WPDSs requires slightly more queries: at most $|Q|$ queries per procedure, where $Q$ is the set of states of $\mathcal{A}_{\text {start }}$. WPDS-version Consider a path $\sigma \in \Delta^{*}$ in the transition relation of a PDS that starts from a configuration $\left\langle p, \gamma_{1} \gamma_{2} \cdots \gamma_{n}\right\rangle$. It can always be decomposed as $\sigma=\sigma_{1} \sigma_{2} \cdots \sigma_{k} \sigma_{k+1}$ (see Fig. 5), such that $\left\langle p, \gamma_{i}\right\rangle \Rightarrow^{\sigma_{i}}\langle p, \varepsilon\rangle$ for $1 \leq i \leq k$ and $\left\langle p, \gamma_{k+1}\right\rangle \Rightarrow^{\sigma_{k+1}}\left\langle p, u_{1} u_{2} \cdots u_{j}\right\rangle$ (or $\sigma_{k+1}$ is empty when $k=n$ ). In other words, $\sigma_{i}, i \leq k$ is the rule sequence whose net effect is to pop off $\gamma_{i}$ without looking at the stack below it, and $\sigma_{k+1}$ is the rule sequence that does not look below $\gamma_{k+1}$ but can replace it and add more symbols on top of the stack. We call the part where symbols are popped $\left(\sigma_{1}, \cdots, \sigma_{k}\right)$ the pop phase and the part where the stack grows $\left(\sigma_{k+1}\right)$, the growth phase.

This property holds because PDS rules can only look at the top of the stack. For $\sigma$ to touch $\gamma_{2}$, it must first pop off $\gamma_{1}$. When it does pop it off, this prefix would be $\sigma_{1}$ (and repeat inductively). If it does not pop off $\gamma_{1}$, then $\sigma$ is already in the growth phase.

We compute poststar $\left(\mathcal{A}_{\text {start }}\right)$ by separating these two phases: First, we compute $\mathcal{A}_{\text {pop }}$ that accepts all configurations reachable after the pop phase. Next, we start from $\mathcal{A}_{\text {pop }}$ and build $\mathcal{A}_{\text {final }}$ that accepts all configurations reachable after the growth phase. The former part is similar to the first phase of the ICFG- 
version of the algorithm, and the latter will correspond to the intraprocedural part (similar to the second phase of the ICFG-version). A running example is shown in Fig. 4(c) $-(f)$.

Terminology (i) A transition $t$ with weight $w$ is added to a weighted automaton $\mathcal{A}$ as follows: if $t$ does not exist in $\mathcal{A}$, then insert it with weight $w$. If it exists in $\mathcal{A}$ with weight $w^{\prime}$, then change its weight to $w^{\prime} \oplus w$. (ii) We say that $\mathcal{A}$ accepts a configuration $c$ with weight at least $w$ if $\mathcal{A}(c) \sqsubseteq w$ (Defn. 2, item 4 ). Note that all configurations are accepted with weight at least $\overline{0}$. (iii) The pop and growth phases are saturation procedures. They convert input $\mathcal{A}$ to output $\mathcal{A}^{\prime}$ by adding transitions to $\mathcal{A}$ until a fixpoint is reached; the fixpoint is the desired output $\mathcal{A}^{\prime}$. Consequently, for all $c, \mathcal{A}^{\prime}(c) \sqsubseteq \mathcal{A}(c)$, and thus for all $c$, $\mathcal{A}_{\text {final }}(c) \sqsubseteq \mathcal{A}_{\text {pop }}(c) \sqsubseteq \mathcal{A}_{\text {start }}(c)$.

Pop Phase Let $w_{\gamma}$ be the weight with which $\gamma$ can be popped, i.e., $w_{\gamma}=$ $\operatorname{IMOP}(\langle p, \gamma\rangle,\langle p, \varepsilon\rangle)=\operatorname{MOP}_{G_{\gamma}}\left[\gamma, x_{\gamma}\right]$, which has been precomputed. We perform saturation on $\mathcal{A}_{\text {start }}$ : if it accepts a configuration $\left\langle p, \gamma \gamma^{\prime} u\right\rangle$, for any $u \in \Gamma^{*}$, with weight $w$, we make it accept $\left\langle p, \gamma^{\prime} u\right\rangle$ with weight at least $w \otimes w_{\gamma}$, and repeat until a fixpoint is reached. This is done as follows: if $\left(p, \gamma, q_{1}\right)$ and $\left(q_{1}, \gamma^{\prime}, q_{2}\right)$ are transitions in the automaton with weight $w_{1}$ and $w_{2}$, respectively, then add the transition $\left(p, \gamma^{\prime}, q_{2}\right)$ with weight $w_{2} \otimes w_{1} \otimes w_{\gamma}$ to the automaton. This process terminates because the number of new transitions added is bounded by $|T|$, where $T$ is the set of transitions of $\mathcal{A}_{\text {start }}$. (This is because a transition $\left(q_{1}, \gamma, q_{2}\right)$ in $\mathcal{A}_{\text {start }}$ can cause at most a single transition $\left(p, \gamma, q_{2}\right)$ to be added to $\mathcal{A}_{\text {pop. }}$.) Defn. 2 (item 4) ensures that weights on them can change at most $\mathcal{H}$ times. Moreover, the running time is bounded by $O_{s}(|T| \mathcal{H})$. Fig. $4(d)$ shows an example: $\mathcal{A}_{\text {start }}$ accepts $\left\langle p, n_{6} n_{3}\right\rangle$ with weight $w_{b} \otimes w_{a}$, the weight with which $n_{6}$ can be popped is $w_{4}$, and $\mathcal{A}_{\text {pop }}$ accepts $\left\langle p, n_{3}\right\rangle$ with weight $w_{b} \otimes w_{a} \otimes w_{4}$.

Growth Phase For the growth phase, we need to consider all configurations reachable from the top symbols of currently accepted configurations, i.e., if $\langle p, \gamma u\rangle, u \in \Gamma^{*}$, is accepted by $\mathcal{A}_{\text {pop }}$ with weight $w$, and $\langle p, \gamma\rangle \Rightarrow^{*}$ $\left\langle p, u^{\prime}\right\rangle, u^{\prime} \in \Gamma^{+}$then $\left\langle p, u^{\prime} u\right\rangle$ should be accepted by $\mathcal{A}_{\text {final }}$ with weight at least $w \otimes \operatorname{IMOP}\left(\langle p, \gamma\rangle,\left\langle p, u^{\prime}\right\rangle\right)$. Now we make use of the observation that called procedures are analyzed independently of their calling context, and reduce this phase to an intraprocedural one. For instance, see Fig. $4(b)$ - the weight $w_{0}$ need not be propagated to transitions involving nodes from bar.

The growth phase proceeds in two parts. The first part constructs automaton $\mathcal{A}_{\text {int }}$ such that if $\mathcal{A}_{\text {pop }}$ accepted configuration $\langle p, \gamma u\rangle$ with weight $w$ and $\langle p, \gamma\rangle \Rightarrow^{*}\left\langle p, \gamma^{\prime}\right\rangle$ then $\mathcal{A}_{\text {int }}$ accepts $\left\langle p, \gamma^{\prime} u\right\rangle$ with weight at least $w \otimes \operatorname{IMOP}\left(\langle p, \gamma\rangle,\left\langle p, \gamma^{\prime}\right\rangle\right)$. This part requires running INTRAQ queries.

For the first part, note that if $\langle p, \gamma\rangle \Rightarrow^{*}\left\langle p, \gamma^{\prime}\right\rangle$, then $\gamma^{\prime}$ must be from the same procedure as $\gamma$ (otherwise, the stack length would be different). Then $\operatorname{IMOP}\left(\langle p, \gamma\rangle,\left\langle p, \gamma^{\prime}\right\rangle\right)=\operatorname{MOP}_{G_{\gamma}}\left[\gamma, \gamma^{\prime}\right]$. Hence, it suffices to do the following: if $(p, \gamma, q)$ is a transition with weight $w$ in $\mathcal{A}_{\text {pop }}$ then add transitions $\left(p, \gamma^{\prime}, q\right)$ to it, for each $\gamma^{\prime}$ in the same procedure as $\gamma$, with weight $w \otimes \operatorname{MOP}_{G_{\gamma}}\left[\gamma, \gamma^{\prime}\right]$. This may add transitions with weight $\overline{0}$ if $\gamma^{\prime}$ is not reachable from $\gamma$, but such transitions can be removed without changing the meaning of a weighted automaton. 
The above process can be optimized. Instead of looking at each transition in isolation, we handle them in bulk. For a state $q$ of $\mathcal{A}_{\text {pop }}$, and a procedure PR, let $S_{q}^{\mathrm{PR}}$ be the set of nodes $s$ in $\mathrm{PR}$ such that $(p, s, q)$ is a transition in $\mathcal{A}_{\text {pop }}$. Let $\mu_{q}^{\mathrm{PR}}$ be such that $\mu_{q}^{\mathrm{PR}}(s)$ is the weight on $(p, s, q)$. Then add transition $\left(p, s^{\prime}, q\right)$ with weight INTRAQ $\left(S_{q}^{\mathrm{Pr}}, \mu_{q}^{\mathrm{PR}}\right)\left(s^{\prime}\right)$. It is easy to see that this imitates the above process, but is more efficient. This results in automaton $\mathcal{A}_{\text {int }}$. The running time is bounded by that required to answer $\mid Q \|$ Proc $\mid$ number of InTRAQ queries, where $Q$ is the set of states of $\mathcal{A}_{\text {pop }}$ (same as those of $\mathcal{A}_{\text {start }}$ ), and |Proc| is the number of procedures in the program.

An example is shown in Fig. 4(e): the algorithm invokes INTRA $Q_{\text {bar }}\left(\left\{n_{6}\right\},\left[n_{6} \mapsto w_{a}\right]\right)$ to add transitions between $p$ and $q$. Next, it invokes INTRA $Q_{\text {foo }}\left(\left\{n_{3}, n_{5}\right\},\left[n_{3} \mapsto w_{b} \otimes w_{a} \otimes w_{4}, n_{5} \mapsto w_{c} \otimes w_{a} \otimes w_{4}\right]\right)$ to add transitions between $p$ and acc.

The second part of the growth phase adds transitions to accept configurations of called procedures. For each procedure PR, add a new state $q_{\mathrm{PR}}$ to $\mathcal{A}_{\text {int }}$, and let CALled (PR) be false initially. Now repeat the following: if $(p, \gamma, q)$ is a transition with weight $w_{1}$ and $\langle p, \gamma\rangle \hookrightarrow\langle p, c r\rangle$ is a WPDS rule with weight $w_{2}$, then $(i)$ if CALLED $\left(\mathrm{PR}_{c}\right)$ is false, then set it to true and add transitions $\left(p, \gamma^{\prime}, q_{\mathrm{PR}_{c}}\right)$ with weight $\mathrm{MOP}_{\mathrm{PR}_{c}}\left[c, \gamma^{\prime}\right]$, for each node $\gamma^{\prime}$ in $\mathrm{PR}_{c} ;(i i)$ add transition $\left(q_{\mathrm{PR}_{c}}, r, q\right)$ with weight $w_{1} \otimes w_{2}$.

The intuition here is that with $\sigma=\langle p, \gamma\rangle \hookrightarrow\langle p, c r\rangle,\langle p, \gamma u\rangle \Rightarrow^{\sigma}\langle p, c r u\rangle$ for any $u \in \Gamma^{*}$. Addition of transitions $\left(p, c, q_{\mathrm{PR}_{c}}\right)$ and $\left(q_{\mathrm{PR}_{c}}, r, q\right)$ ensures that the latter configuration is accepted (with appropriate weights). Next, $c$ can reach node $\gamma^{\prime}$ in the same procedure with weight $\mathrm{MOP}_{\mathrm{PR}_{c}}\left[c, \gamma^{\prime}\right]$, for which the transitions $\left(p, \gamma^{\prime}, q_{\mathrm{PR}_{c}}\right)$ are added. Note that the weight at the call site $\left(w_{1} \otimes w_{2}\right)$ gets stored on the transition $\left(q_{\mathrm{PR}_{c}}, r, q\right)$. Thus, $\mathrm{PR}_{c}$ is analyzed independently of this weight and the weights on transitions $\left(p, \gamma^{\prime}, q_{\mathrm{PR}_{c}}\right)$, for each $\gamma^{\prime}$ in $\mathrm{PR}_{c}$, are independent of the input query.

This process terminates because only a finite number of states are added. The trick of bounding the number of states is common in reachability algorithms for PDSs $[18,21]$. The running time is bounded by $O_{s}(|\operatorname{Ret}||Q|)+O(|\Gamma|)$, where Ret is the set of return sites in the program. This running time is subsumed by that of the first part. Fig. $4(f)$ shows an example.

Complexity First, we discuss the complexity of solving a query after preprocessing has been completed. Let $Q$ be the set of states of $\mathcal{A}_{\text {start }}$, and $T$ the set of its transitions. The pop phase has running time $O_{s}(|T| \mathcal{H})$. The growth phase, when using Alg3 for InTRAQ queries, has running time $O_{s}(|Q||\operatorname{Proc} \|| E|\log | V \mid)$, where $\mid$ Proc $\mid$ is the number of procedures in the program, and $|E|$ and $|V|$ are the average number of nodes per procedure. This gives a total worst-case running time of $O_{s}(|T| \mathcal{H}+|Q| \mid$ Proc ||$E|\log | V \mid)$. The number of nodes per procedure usually remains constant even as program size increases. Treating $\log |V|$ as a constant, and writing $|E| \mid$ Proc $\mid$ as $|\Delta|$ (the number of WPDS rules), we get a total complexity of $O_{s}(|T| \mathcal{H}+|Q||\Delta|)$. This is asymptotically better than the complexity of previous algorithms $[18,9]$, which is $O_{s}(|T| \mathcal{H}+(|Q|+\mid$ Proc $\mid)|\Delta| \mathcal{H})$ 
in each case. Note the reduced dependence on $\mathcal{H}$ for our algorithm (hence less fixpoint computation around loops and recursion).

If the initial set of configurations is finite (i.e., automaton $\mathcal{A}_{\text {start }}$ does not have any cycles), the running time of the pop phase can be bounded by $O_{s}(|T|)$, resulting in a total running time (after preprocessing) that is completely independent of the height of the weight domain (which is not true for any other WPDS reachability algorithm).

The complexity for preprocessing is dominated by the step that computes procedure summaries (and path sequences as a by-product). This just requires a single dataflow query, whose complexity is $O_{s}(|\operatorname{Proc}||\Delta| \mathcal{H})$ [9]. Using path sequences to compute the other preprocessing information is fairly quick.

\section{Experiments}

We refer to the implementation of the algorithm in this paper as SWPDS (Summary-WPDS). We compare against saturation-based [18] and optimized [9] approaches for solving WPDS queries, of which we pick the better running time and refer to it as OWPDS (Old-WPDS).

We carried out experiments on WPDSs obtained from three different applications. The first application is affine-relation analysis (ARA) of x86 programs [1]. A WPDS is produced from the $\mathrm{x} 86$ program using the weight domain for ARA described in [12]. The goal is to discover affine relationships (linear equalities) between machine registers.

The first experiment is to find out loop invariants (where loops are discovered by Bourdoncle's decomposition technique [3]). For outermost loops in a procedure, a loop summary is obtained as the weight $\operatorname{IMOP}(\langle p$, head $\rangle,\langle p$, head $\rangle)$, where head is the head of the loop. This can be calculated by computing $\mathcal{A}=\operatorname{poststar}(\{(\langle p$, head $\rangle, \overline{1})\})$, and $\mathcal{A}(\langle p$, head $\rangle)$. Loop invariants can be calculated easily from these summaries. These invariants give the conditions that hold at the head of the loop and are re-established after each iteration of the loop. We use common Windows executables, including code for the called libraries, and ran the experiments on a $3.2 \mathrm{GHz}$ P4 processor with 3.3GB RAM running Windows XP.

A conventional way to solve these queries would be to compute the procedure summaries, plug them at the call-sites and then solve each loop as an intraprocedural problem. We call this technique OWPDS2. It uses ALG1 to solve each loop. Tab. 1 reports the following timings: the time taken to answer each query independently (OWPDS); the time taken by OWPDS2 (after procedure summaries have been computed); the preprocessing time for SWPDS (Setup); and the time taken to answer all queries using SWPDS, after preprocessing. We make two comparisons: (SWPDS+Setup) versus OWPDS, for which we are about 17 times faster (not shown in the table), and SWPDS versus OWPDS2, for which we are 1.5 times faster. We do not take the setup times into account in the second comparison because SWPDS preprocessing only computes procedure summaries (other preprocessing is unnecessary for this application). 


\begin{tabular}{|c|c|c|c|c|c|c|c|c|c|c|c|c|c|c|c|}
\hline & & & & \multicolumn{4}{|c|}{ Time (s) } & & \multicolumn{3}{|c|}{ Constant Registers } & \multicolumn{4}{|c|}{ Other Invariants } \\
\hline Prog & Insts & Procs & Loops & \begin{tabular}{|l|} 
OWPDS \\
\end{tabular} & OWPDS2 & Setup & SWPDS & Speedup & 0 & \begin{tabular}{|l|l|}
$1-3$ & $4-6$ \\
\end{tabular} & $7-8$ & 0 & & $2 \mid$ & $\geq 3$ \\
\hline latex & 63711 & 609 & 280 & 168 & 5.7 & 28 & 4.3 & 1.3 & 53 & \begin{tabular}{l|l|}
44 & 152
\end{tabular} & 31 & 124 & 125 & 31 & 0 \\
\hline attrib & 103473 & 964 & 537 & 290 & 8.3 & 46 & 5.1 & 1.7 & 97 & 114271 & 55 & 227 & 254 & 56 & 0 \\
\hline $\mathrm{ftp}$ & 130352 & 1271 & 634 & 731 & 13.5 & 26 & 8.7 & 1.6 & 130 & $|126| 320$ & 58 & 290 & 280 & 64 & 0 \\
\hline notepad & 167430 & 1609 & 749 & 597 & 12.1 & 43 & 8.2 & 1.5 & 162 & $\mid$\begin{tabular}{l|l|}
1569 \\
\end{tabular} & 62 & 325 & 336 & 87 & 1 \\
\hline $\mathrm{cmd}$ & 192579 & 1783 & 869 & 3415 & 24.1 & 64 & 18.0 & 1.3 & 256 & 156391 & 66 & 431 & 355 & 80 & 3 \\
\hline
\end{tabular}

Table 1. ARA experiments. The speedup is reported for SWPDS versus OWPDS2.

\begin{tabular}{|c|c|c|c|c|c|c|c|c|c|c|c|c|}
\hline & & & & For & ward Rea & ach. & Bacl & kward Rea & ach. & & CBMC & \\
\hline Prog & Nodes & Procs & Setup & SWPDS & OWPDS & Speedup & SWPDS & OWPDS & Speedup & SWPDS & OWPDS & Speedup \\
\hline bugs5 & 36971 & 291 & 11.9 & 4.2 & 18.4 & 4.4 & 1.4 & 8.4 & 5.8 & 98 & 183 & 1.7 \\
\hline unified-serial & 38234 & 291 & 15.4 & 5.3 & 24.5 & 4.7 & 1.7 & 12.1 & 7.1 & 129 & 238 & 1.6 \\
\hline slam & 7161 & 97 & 3.5 & 2.7 & 14.2 & 5.3 & 0.4 & 2.1 & 6.0 & 87 & 115 & 1.3 \\
\hline iscsiprt1 & 4803 & 82 & 0.6 & 0.27 & 0.84 & 3.1 & 0.06 & 0.36 & 6.0 & 6.3 & 12 & 1.7 \\
\hline ufloppy 13 & 5679 & 64 & 1.5 & 0.6 & 2.0 & 3.1 & 0.07 & 0.7 & 10.3 & 12 & 20 & 1.5 \\
\hline
\end{tabular}

Table 2. Experiments on Boolean programs: (i) Forward and backward reachability from the set of configurations $n R e t^{*}$. The node $n$ was chosen randomly, and running times, reported in seconds, were averaged across 5 queries. The speedup is reported per query, ignoring the setup time. (ii) Simulated CBMC queries. The speedup reported takes the setup time into account.

Tab. 1 also shows a distribution of the obtained loop invariants. Loop invariants that indicate that a register remains unchanged after each loop iteration (even though it may get modified inside the loop) are reported separately from other kinds of invariants. The last eight columns show the number of loops that have a certain number of constant registers or affine invariants. For example, in latex, 53 loops do not have any constant registers, and 31 loops have 2 linearly independent invariants. These invariants can be beneficial to other analysis (e.g., they identify loop-induction variables).

The second application is Boolean program verification using Moped [21]. Boolean programs are converted to WPDSs, and dataflow analysis is used for proving properties of the program. In our experiments, the Boolean programs were obtained as a result of predicate abstraction. The following experiments were run on a $3 \mathrm{GHz}$ P4 processor with 2GB RAM running Linux, and the results are reported in Tab. 2.

We ran queries starting from the set of configurations $\left(n \operatorname{Ret}^{*}\right)$, where $n$ is a program node and Ret is the set of return-site nodes. Such queries are useful for finding out the net effect in going from one program statement to another (for finding dependencies between the two). After the setup time, SWPDS was 4 times than OWPDS on forward reachability queries, and 7 times faster on backward reachability (our algorithm for solving backward reachability is given in [10]). This experiment also shows that two dataflow queries are enough to recover the SWPDS preprocessing time.

The third application (also based on Moped, running on the same Linux platform), considers context-bounded model checking (CBMC) [13], which aims to find all reachable states of a concurrent program under a bound on the number of context switches. Because we lack a front-end to abstract concurrent programs, 
we performed simulated experiments on sequential programs. We assume that the global variables of the program are shared with an environment that can randomly change their value, and the environment itself does not possess any local state. We ran one branch of CBMC along which control is transferred to the environment 5 times. Essentially, this requires the following: for random global states $g_{1}, \cdots, g_{5}$, if $\mathcal{A}_{0}$ describes the initial configuration of the (sequential) program, then compute $\mathcal{A}_{1}=\operatorname{poststar}\left(\mathcal{A}_{0}\right)$ and $\mathcal{A}_{i+1}=\operatorname{poststar}\left(\operatorname{Modify}\left(\mathcal{A}_{i}, g_{i}\right)\right)$ for $i=1$ to 5 . Here, $\operatorname{Modify}(\mathcal{A}, g)$ is an automaton that represents the same set of states as $\mathcal{A}$, but with the shared state changed to $g$. Because the result of running poststar is a bigger automaton than the original one, we report the total time taken to run all the queries. The average speedup was 1.6 times.

The varying amount of speedups in the different applications seems to be related to the size of $S$ in IntraQ $(S, \mu)$ queries. For the ARA experiments, $|S|$ was always 1 (maximum speedup over OWPDS); for the second application, $S$ was usually the set of return sites in a procedure; for CBMC, $S$ consisted of all nodes in a procedure (least amount of speedup). Worst-case complexity does not predict this effect; this is an observation about measured behavior.

\section{Related Work}

The goal of incremental program analysis $[4,14,11,20,5]$ is to reuse as much information as possible from previous fixpoint computations to calculate a new fixpoint when a small change is made to the program. Our work has aspects that resemble incremental computing in that we avoid recomputing the same information in response to changes in the query. However, we have a single preprocessing step to compute summaries and path sequences; this information is used by multiple dataflow queries, but there is no additional information tabulated during one query for use by a later query. This is because we do not look into the weights (and avoid caching computations over them), and base our optimizations only on the program control structure.

Another closely related category of work is that on demand-driven dataflow analysis $[15,19,6]$. There the focus is to do only as much work as is required to solve a query, and not redo it in a subsequent query. However, these techniques assume a particular form for the weights, and do look inside them (to work with exploded $C F G s$ ). We make fewer assumptions about the weights. These techniques would not be applicable to the weight domain we considered in our first application or be able to work with BDDs, as required by the other applications.

Technically, the most closely related piece of work is our previous work on speeding up a single dataflow query [9] called FWPDS. It used Tarjan's algorithm at the intraprocedural level to compute regular expressions for solving MOP values, and combined it with techniques like incremental computation of regular expressions to extend it for interprocedural analysis. In this paper, we use Tarjan's algorithm to compute path sequences. We combine it with a new WPDS reachability algorithm that shows how to summarize and reuse information at the interprocedural level. Moreover, FWPDS required the starting set of 
configurations to be in hand before it built the graphs on which it ran Tarjan's algorithm and may build different graphs for different queries, preventing it from sharing information between them. SWPDS outperforms FWPDS (included as OWPDS in $\S 5)$.

\section{References}

1. G. Balakrishnan and T. Reps. Analyzing memory accesses in x86 executables. In $C C, 2004$.

2. A. Bouajjani, J. Esparza, and T. Touili. A generic approach to the static analysis of concurrent programs with procedures. In POPL, 2003.

3. F. Bourdoncle. Efficient chaotic iteration strategies with widenings. In FMPA, 1993.

4. J. Cai and R. Paige. Program derivation by fixed point computation. SCP, 11(3), 1989.

5. C. L. Conway, K. S. Namjoshi, D. Dams, and S. A. Edwards. Incremental algorithms for inter-procedural analysis of safety properties. In $C A V, 2005$.

6. E. Duesterwald, R. Gupta, and M. L. Soffa. Demand-driven computation of interprocedural data flow. In POPL, 1995.

7. S. Graham and M. Wegman. A fast and usually linear algorithm for global flow analysis. J. ACM, 23(1), 1976.

8. J. Knoop and B. Steffen. The interprocedural coincidence theorem. In $C C, 1992$.

9. A. Lal and T. Reps. Improving pushdown system model checking. In $C A V, 2006$.

10. A. Lal and T. Reps. Solving multiple dataflow queries using WPDSs. Technical Report 1632, University of Wisconsin-Madison, Mar. 2008.

11. Y. A. Liu, S. D. Stoller, and T. Teitelbaum. Static caching for incremental computation. TOPLAS, 20(3), 1998.

12. M. Müller-Olm and H. Seidl. Analysis of modular arithmetic. In ESOP, 2005.

13. S. Qadeer and J. Rehof. Context-bounded model checking of concurrent software. In $T A C A S, 2005$.

14. G. Ramalingam and T. W. Reps. A categorized bibliography on incremental computation. In $P O P L, 1993$.

15. T. Reps. Solving demand versions of interprocedural analysis problems. In $C C$, 1994.

16. T. Reps, S. Horwitz, and M. Sagiv. Precise interprocedural dataflow analysis via graph reachability. In POPL, 1995.

17. T. Reps, A. Lal, and N. Kidd. Program analysis using weighted pushdown systems. In FSTTCS, 2007.

18. T. Reps, S. Schwoon, S. Jha, and D. Melski. Weighted pushdown systems and their application to interprocedural dataflow analysis. In $S C P$, volume 58, 2005.

19. S. Sagiv, T. Reps, and S. Horwitz. Precise interprocedural dataflow analysis with applications to constant propagation. Theor. Comput. Sci., 167(1\&2), 1996.

20. D. Saha and C. R. Ramakrishnan. Incremental evaluation of tabled logic programs. In $I C L P, 2003$.

21. S. Schwoon. Model-Checking Pushdown Systems. PhD thesis, Technical Univ. of Munich, Munich, Germany, July 2002.

22. M. Sharir and A. Pnueli. Two approaches to interprocedural data flow analysis. In Program Flow Analysis: Theory and Applications. Prentice-Hall, 1981.

23. R. E. Tarjan. Fast algorithms for solving path problems. J. ACM, 28(3):594-614, 1981. 